\title{
Estudo microscópico de páprica (Capsicum annuum L.): detecção de fraudes e matérias estranhas
}

\author{
Microscopic study of paprika (Capsicum annuum L.): detection of \\ fraud and foreign matter
}

\section{Laís Fernanda de \\ Pauli-Yamadal,* (iD}

Cinthia lara de Aquino"

Augusta Mendes da Silva' (iD)

Maria Aparecida Moraes Marciano'

Elaine Cristina de Mattos"II ID

Márcia Dimov Nogueira' iD
Núcleo de Morfologia e Microscopia, Centro de Alimentos, Instituto Adolfo Lutz Central, São Paulo, SP, Brasil

" Centro de Laboratório Regional de Ribeirão Preto, Instituto Adolfo Lutz, Ribeirão Preto, SP, Brasil

III Centro de Laboratório Regional de Santo André, Instituto Adolfo Lutz, Santo André, SP, Brasil

\section{* E-mail: lais.yamada@ial.sp.gov.br}

Recebido: 29 nov 2019 Aprovado: 24 jul 2020

\section{RESUMO}

Introdução: A páprica (Capsicum annuum L.) é um condimento utilizado mundialmente, produzido a partir de diversas variedades de pimentão maduro, seco e moído e está sujeita a adulterações e fraudes, além de contaminações decorrentes de falhas nas boas práticas de produção, armazenamento ou na comercialização. Objetivo: Avaliar a autenticidade e a presença de matérias estranhas em pápricas comercializadas no estado de São Paulo, assim como verificar a sua conformidade em relação à legislação nos parâmetros microscópicos. Método: Foram analisadas 43 amostras de páprica para verificação da autenticidade e para a pesquisa de matérias estranhas, utilizando método preconizado nos Métodos Oficiais da Association of Official Analytical Collaboration International. Resultados: A avaliação da autenticidade evidenciou fraude em $30,0 \%$ das amostras. 0 ingrediente adicionado com maior frequência foi o amido de Zea mays L. (milho), acompanhado principalmente de Bixa orellana L. (urucum). Entre as matérias estranhas detectadas, os fragmentos de pelo de roedor e os fragmentos de insetos foram os mais frequentes, presentes em $91,0 \%$ e $79,0 \%$ das amostras, respectivamente. As amostras puras apresentaram maior quantidade de fragmentos de pelo de roedor, enquanto as amostras adulteradas, maior quantidade de fragmentos de insetos. Em relação à legislação sanitária, 88,0\% das amostras foram consideradas insatisfatórias, destas, 30,0\% por estarem adulteradas e $77,0 \%$ por apresentarem quantidade de matérias estranhas acima do limite tolerado. Conclusões: Os resultados deste trabalho revelaram fraude e falhas nas boas práticas na cadeia produtiva da páprica, ressaltando a necessidade de intensificação da vigilância desse tipo de produto com vistas à garantia de sua oferta à população de forma fidedigna e segura.

PALAVRAS-CHAVE: Capsicum; Fraude; Sujidades Leves; Pelos de Roedor; Segurança de Alimentos

\section{ABSTRACT}

Introduction: Paprika (Capsicum annuum L.) is a condiment used worldwide; it is produced from various varieties of ripe, dried and ground peppers, and it is subject to adulteration and fraud, as well as to contamination due to failures in good practices of production, storage or marketing. Objective: To evaluate the authenticity and the presence of foreign matter in paprika samples marketed in the state of São Paulo, as well as to verify their compliance with the legislation on microscopic parameters. Method: Forty-three paprika samples were analyzed to verify the authenticity and to search foreign matter using the method recommended by the Official Methods of the Association of Official Analytical Collaboration International. Results: The authenticity evaluation showed fraud in $30.0 \%$ of the samples. The most frequently added ingredient was Zea mays L. (corn) starch, accompanied mainly by Bixa orellana L. (annatto). Among the foreign matter detected, rodent hair fragments and insect fragments were the most frequent, present in $91.0 \%$ and $79.0 \%$ of the samples, respectively. Pure samples presented larger amount of rodent hair fragments, while adulterated samples larger amount of insect fragments. Regarding the sanitary legislation, $88.0 \%$ of the samples were considered unsatisfactory; of these, $30.0 \%$ for being adulterated and $77.0 \%$ for presenting quantity of foreign matter above the tolerated limit. Conclusions: 
The results of this work revealed fraud and failures in good practices in the paprika's productive chain, emphasizing the need for intensified surveillance of this type of product in order to guarantee its reliable and safe supply to the population.

KEYWORDS: Capsicum; Fraud; Light Filth; Rodent Hair; Food Safety

\section{INTRODUÇÃO}

A páprica (Capsicum annuum L.), conhecida também por colo$\mathrm{rau}^{1}$, é um condimento utilizado mundialmente, produzido a partir de diversas variedades de pimentão maduro, seco e moído, empregado principalmente como corante natural vermelho ${ }^{2,3}$.

As pápricas podem ser doces, utilizadas como corante, ou picantes, com níveis de ardume variáveis, e são usadas como flavorizantes para realçar o sabor dos alimentos. A demanda por corantes naturais para a alimentação humana vem aumentando, principalmente em substituição aos corantes sintéticos, cujo uso em alimentos vem sendo restringido em alguns países europeus ${ }^{2,4}$.

Assim como outras especiarias, como o açafrão e a pimenta preta moída, a páprica está sujeita a adulterações e fraudes, constando entre o rol dos 25 alimentos com maior número de registros de fraudes. Essas podem ocorrer por meio de adição ou substituição de substâncias ou ingredientes, geralmente por motivações econômicas, visando o aumento da lucratividade por meios ilícitos. A detecção dessas fraudes é um desafio constante e estima-se que são grandes os prejuízos decorrentes dessas adulterações ${ }^{5,6}$.

As especiarias e ervas, apesar de consumidas em pequenas quantidades, preocupam os profissionais ligados à segurança de alimentos, devido ao seu uso como tempero em uma grande diversidade de alimentos, muitas vezes sem cozimento posterior. O longo período de produção e as cadeias comerciais complexas aumentam as possibilidades de contaminações naturais, acidentais ou intencionais. Há relatos em todo o mundo sobre a contaminação química, microbiológica, fraude e/ou adulteração de páprica ${ }^{7}$.

Há registros da produção de páprica na região Sudeste do Brasil, principalmente em Minas Gerais ${ }^{3}$, porém atualmente os dados nacionais de fabricação desse condimento são escassos. Segundo a plataforma Comex Stat $^{8}$, banco de dados do Ministério da Indústria, Comércio Exterior e Serviços, entre 2017 e 2018, o Brasil importou cerca de 2.000 t de pimentas do gênero Capsicum, trituradas ou em pó e $1.500 \mathrm{t}$ de pimentas inteiras, sendo a China o principal fornecedor, seguida da Índia.

Para a produção da páprica, após a colheita, os pimentões de coloração vermelha intensa são selecionados, higienizados, desidratados e moídos. Fatores como temperatura, umidade, condições de secagem e de armazenamento influenciam a qualidade do produto, podendo causar oxidação, degradação dos pigmentos e favorecer o crescimento de bactérias e fungos, caso não sejam controlados $4,9,10$.

Somente o estudo de Correia et al. ${ }^{11}$ foi reportado no Brasil sobre a qualidade sanitária quanto à presença de matérias estranhas em páprica, sendo escassos os dados nacionais sobre este produto.
A conformidade dos alimentos quanto a sua autenticidade é baseada na Resolução da Diretoria Colegiada (RDC) da Agência Nacional de Vigilância Sanitária (Anvisa) $n^{\circ} 259$, de 20 de setembro de $2002^{12}$, que regulamenta a rotulagem de alimentos embalados. Como referência para a avaliação da presença de matérias estranhas é utilizada a RDC Anvisa $n^{\circ} 14$, de 28 de março de $2014^{13}$, que estabelece limites de tolerância para alguns alimentos, dentre eles a páprica.

O objetivo desse estudo foi avaliar a autenticidade quanto à identidade histológica e a qualidade sanitária quanto à presença de matérias estranhas em amostras de páprica comercializadas em diversos municípios do estado de São Paulo, assim como verificar a sua conformidade em relação à legislação nos parâmetros microscópicos.

\section{MÉTODO}

Foram analisadas 43 amostras de páprica no Núcleo de Morfologia e Microscopia do Instituto Adolfo Lutz (IAL), Laboratório Central de São Paulo e Centro de Laboratório Regional de Santo André, provenientes das Vigilâncias Sanitárias, em atendimento ao Programa Paulista de Análise Fiscal de Alimentos em 2017, 2018 e 2019.

Tipos diferentes de páprica disponíveis no comércio foram analisados, independente da denominação expressa no rótulo, páprica picante, doce, doce defumada, defumada e com pimenta calabresa, de 16 marcas distintas com lotes e prazos de validade diferentes.

A autenticidade do produto foi avaliada por meio do isolamento dos elementos histológicos presentes, conforme técnica descrita nos Métodos de Análise Microscópica de Alimentos ${ }^{14}$ e identificação, de acordo com Oliveira et al. ${ }^{15}$. Para a pesquisa de matérias estranhas foi aplicado o método 977.25B (16.14.22), de extração de sujidades leves em páprica, preconizado nos Métodos Oficiais da Association of Official Analytical Collaboration (AOAC) International ${ }^{16}$ e recomendado pela RDC Anvisa $n^{0} 14 / 2014^{13}$. Os pelos animais foram identificados segundo Teerink ${ }^{17}$.

A análise dos dados foi realizada no programa Microsoft Excel 2010, aplicando-se o teste qui-quadrado com nível de significância de 95\%.

\section{RESULTADOS E DISCUSSÃO}

$\mathrm{Na}$ avaliação da autenticidade, a identificação de elementos histológicos evidenciou fraude em $30,0 \%$ das amostras, com adição de diferentes componentes, além do pimentão. 
Entre as amostras adulteradas, o amido de milho foi o ingrediente adicionado com maior frequência (85,0\%). Em 46,0\% delas foi observada a presença concomitante de Bixa orellana L. (urucum) e do amido de Zea mays L. (milho), as demais, além do pimentão, revelaram a presença de urucum e amido alterado (7,7\%); Piper nigrum L. (pimenta do reino) (7,7\%); amido de milho, pimenta do reino, Curcuma longa L. (cúrcuma) e Zingiber officinale Roscoe (gengibre) $(7,7 \%)$ e amido de milho e vegetal não identificado $(7,7 \%)$. A associação de amido de milho e urucum, denominada colorífico ${ }^{1}$, apresenta grande semelhança visual à páprica, favorecendo a fraude e prejudicando sua detecção pelos consumidores.

De acordo com o Regulamento Técnico para Rotulagem de Alimentos Embalados, RDC Anvisa $n^{\circ} 259 / 2002^{12}$, com exceção de alimentos com um único ingrediente, os componentes do alimento devem estar declarados na lista de ingredientes contida no rótulo. Essa norma é utilizada para verificação da conformidade dos alimentos em relação à sua identidade, portanto, nesse estudo, todas as amostras adulteradas estavam insatisfatórias por não estarem condizentes com a denominação de venda "páprica" ou por apresentarem vegetais não declarados na lista de ingredientes.

A análise histológica realizada é uma análise qualitativa, que possibilita a identificação dos vegetais presentes na amostra e a detecção de fraudes, porém não permite quantificá-los, não sendo possível determinar a quantidade dos ingredientes adicionados nas amostras analisadas.

Geralmente as motivações das fraudes alimentares são econômicas e podem envolver fabricantes, varejistas ou importadores, com o intuito de obter ganhos monetários ilícitos. Há registros de fraudes alimentares a milhares de anos, que, por na maioria das vezes não ocasionarem danos à saúde e não causarem nitidamente um problema de qualidade nos produtos, não são percebidos pelo consumidor. Porém, a adição de ingredientes não declarados, geralmente de valor e de qualidade inferiores ao produto, além de causar danos econômicos ao consumidor, pode ter consequências à saúde, como no caso de determinadas alergias ou intolerâncias alimentares ${ }^{6}$.
Das 43 amostras de páprica analisadas, apenas uma não apresentou matérias estranhas, sendo que os fragmentos de pelo de roedor e os fragmentos de insetos foram os mais frequentes, presentes em $91,0 \%$ e $79,0 \%$ das amostras, respectivamente (Figura 1).

Os fragmentos de pelo isolados das amostras eram diminutos e tinham as extremidades aparentemente cortadas, como se tivessem sido triturados junto ao produto, denotando uma contaminação prévia ao processo de moagem. Estes fragmentos eram muito semelhantes entre si e continham o padrão medular compatível com o padrão de pelos de roedor, entre eles de camundongo (Mus musculus L.) ${ }^{17}$ (Figura 2). A presença de fragmentos de pelo de roedor, na frequência e quantidade encontradas, pode ser decorrente de falhas na higienização da matéria prima ou infestação de roedores durante a secagem.

Correia et al. ${ }^{11}$ avaliaram a presença de matérias estranhas em pápricas comercializadas no estado de São Paulo e obtiveram resultados semelhantes aos do presente estudo, porém em frequência e quantidade de matérias estranhas diferentes, estando os pelos de roedor presentes em $34,0 \%$ das amostras, os fragmentos de insetos em $100 \%$ e os ácaros em 12,5\%.

Os pelos ou fragmentos de pelos de roedor são uma matéria estranha indicativa de risco à saúde humana por serem potenciais veiculadores de patógenos para os alimentos ${ }^{13}$, evidenciando que 0 roedor entrou em contato com o produto em alguma etapa do processamento, propiciando a contaminação do alimento por meio de suas excreções. $O$ consumo do condimento contendo essa matéria estranha sem cozimento prévio pode agravar esse risco.

Os roedores são a principal praga da plantação de pimentão, pois danificam os frutos durante o seu amadurecimento mais do que os consomem, levando à sua queda ou à diminuição da qualidade. Estima-se que os danos causados por roedores podem chegar a $30 \%$ do rendimento total de pimentões, afetando a quantidade e a qualidade da páprica produzida. Em um trabalho desenvolvido em Moçambique para verificar as consequências decorrentes do ataque de roedores em plantações de pimentão, verificou-se que, nos campos onde foram colocadas armadilhas para captura dos roedores, houve uma redução de $49 \%$

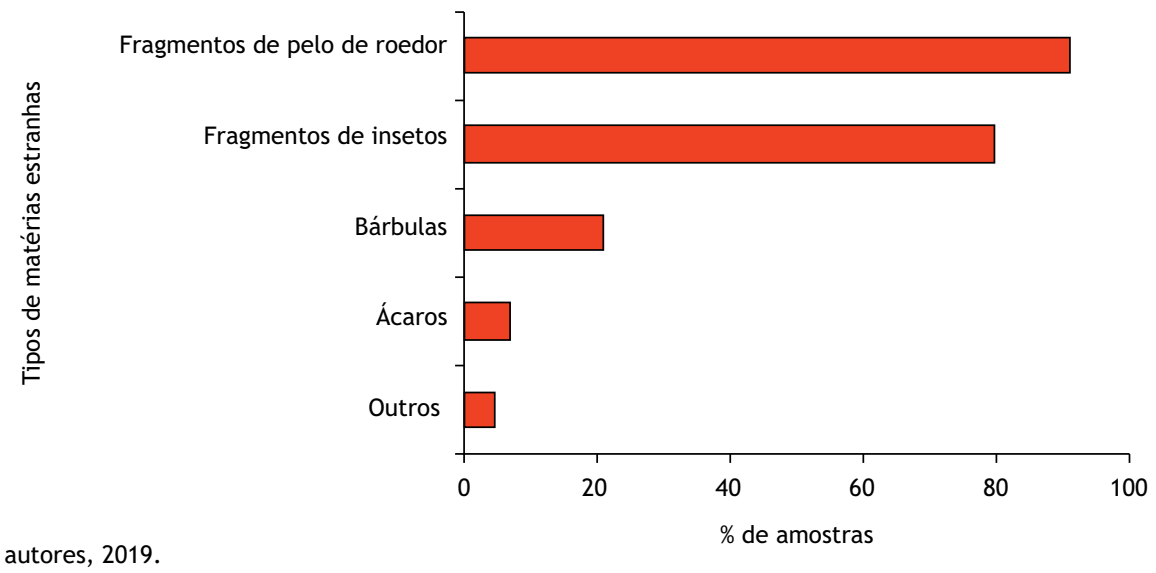

Fonte: Elaborada pelos autores, 2019

Figura 1. Frequência relativa da presença de matérias estranhas nas amostras de páprica analisadas no período de 2017 a 2019. 


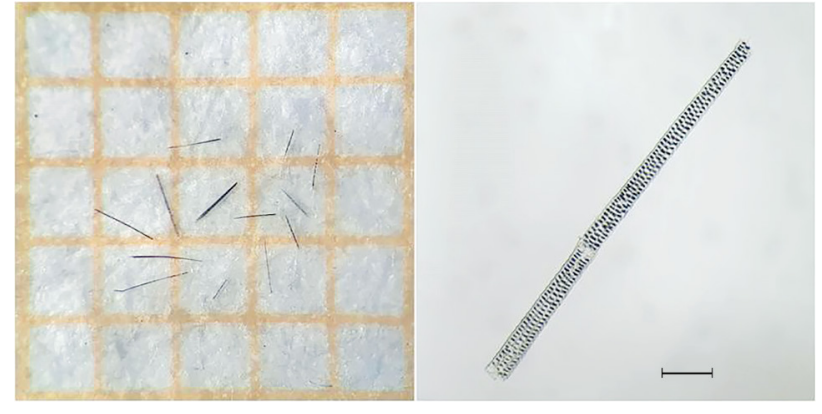

Fonte: Elaborada pelos autores, 2019.

Figura 2. Fragmentos de pelos de roedor isolados de amostra de páprica $(A)$ vistos em microscópio estereoscópico sobre papel milimetrado e (B) em microscópio óptico evidenciando o detalhe do padrão medular de Mus musculus L.

nas perdas de produção, assim como melhorou a qualidade dos pimentões colhidos ${ }^{18}$.

Os fragmentos de insetos detectados em maior quantidade nas amostras adulteradas do que nas puras permitem sugerir que parte deles pode ter sido carreada pelos ingredientes adicionados à páprica, como o amido de milho. A grande quantidade de fragmentos de insetos em fubás já havia sido reportada por Atuí et al. ${ }^{19}$, sendo $79,0 \%$ das amostras analisadas consideradas insatisfatórias pela legislação em vigor na época, dentre elas, $75,3 \%$ continham larvas, sugerindo a produção do alimento com grãos de milho infestados internamente. Dupchak ${ }^{20}$ observou que, quanto maior a infestação de grãos, maior o número de fragmentos de insetos no produto final e pior a qualidade do produto.

Os fragmentos de insetos são considerados matérias estranhas indicativas de risco à saúde humana se forem provenientes de baratas, formigas ou moscas, ou podem ser indicativos de falhas das boas práticas se forem originários de artrópodes próprios da cultura e do armazenamento ${ }^{13}$. Apesar de não terem sido identificados quanto à espécie, os fragmentos isolados neste estudo não apresentavam características morfológicas de artrópodes considerados de risco à saúde.

Entre as amostras, 21,0\% apresentaram bárbulas, sendo que uma delas apresentou quantidade incontável dessa matéria estranha na alíquota analisada. As bárbulas são parte das ramificações das penas de aves e sua presença nos alimentos indica problemas com infestação ou invasão dos locais de produção por aves que podem atuar como pragas e transportar diversos micro-organismos, inclusive patógenos, como fungos, parasitos e bactérias ${ }^{21}$. A identificação específica das bárbulas encontradas não foi realizada, porém qualquer fragmento de pena nos alimentos constitui contaminação e sua presença reforça os indícios de falha das boas práticas em alguma etapa da produção. A legislação brasileira considera bárbula de pombo como uma matéria estranha de risco à saúde ${ }^{13}$.

Os ácaros estavam presentes em 7,0\% das amostras, com contagem máxima de dez. Resultados semelhantes foram observados por Correia et al. ${ }^{11}$, em que $12,5 \%$ das amostras apresentaram ácaros com número máximo de 34 em uma única amostra. Os ácaros representam matéria estranha indicativa de falhas das boas práticas e podem estar associados a problemas no armazenamento, umidade, presença de fungos, entre outros fatores ${ }^{22}$.

Entre as outras matérias estranhas encontradas, foi detectado um pelo inteiro de animal não identificado em uma amostra e fibras sintéticas em outra.

As condições de produção, armazenamento e transporte da páprica podem tornar o produto vulnerável a diversas infestações. A distância e o tempo decorrido entre a produção e o consumo também podem favorecer essas contaminações. 0 cultivo em áreas controladas, assim como a utilização de tecnologias de secagem, como secadores solares ou elétricos podem evitar contaminações externas, comuns durante a secagem natural ao sol ${ }^{9}$.

No presente estudo, 296 fragmentos de pelo de roedor chegaram a ser isolados de uma única amostra (Tabela 1), quantidade muito superior à de Correia et al. ${ }^{11}$, cujo número máximo foi de 9,5, já em relação aos fragmentos de insetos, foram encontradas quantidades aproximadas de 280 neste trabalho e de 256 no estudo de Correia et al. ${ }^{11}$. Apesar de terem sido encontradas elevadas contagens de fragmentos de pelo de roedor e de fragmentos de insetos em algumas amostras, os valores das medianas ficaram em torno de 26,0 e 13,0, respectivamente. Para pelo de roedor, este valor é bastante elevado em comparação ao estudo de Correia et al. ${ }^{11}$, cuja mediana foi 0,00 , porém, quanto aos fragmentos de insetos, a mediana obtida neste estudo foi inferior à de Correia et al. ${ }^{11}$, que apresentou o valor de 25,75.

Foi observada uma associação estatística $(p<0,05)$ entre a autenticidade das amostras e a presença de matérias estranhas acima do limite tolerado pela legislação, evidenciando que as amostras puras apresentaram maior quantidade de matérias estranhas.

Ao se comparar as amostras puras com as adulteradas, percebe-se que as puras apresentaram em média 64 fragmentos de pelos de roedor e 18 fragmentos de insetos, enquanto as adulteradas continham em média 22 fragmentos de pelos de roedor e 66 fragmentos de insetos. Esses resultados possibilitam sugerir que as contaminações de pelos de roedor advêm das amostras autênticas e a adição de outros ingredientes pode ter diluído

Tabela 1. Estatística descritiva da contagem de matérias estranhas detectadas em amostras de páprica, no estado de São Paulo, em $2017-2019$.

\begin{tabular}{lcccc} 
& Fragmento de pelo de roedor & Fragmentos de insetos & Ácaro & Bárbula \\
\hline Média & 51,3 & 31,9 & 0,6 & 0,5 \\
Mediana & 26,0 & 13,0 & 0,0 & 0,0 \\
Mínimo & 0,0 & 0,0 & 0,0 & 0,0 \\
Máximo & 296,0 & 280,0 & 10,0 & incontáveis \\
\hline
\end{tabular}

Fonte: Elaborada pelos autores, 2019. 
Tabela 2. Distribuição das amostras de páprica analisadas no Instituto Adolfo Lutz em 2017-2019, em relação à quantidade de matérias estranhas detectadas, conforme limites de tolerância estabelecidos pela RDC n 14/2014.

\begin{tabular}{|c|c|c|c|}
\hline \multirow{2}{*}{ Matérias estranhas } & Ausente & Dentro do limite & Acima do limite \\
\hline & \multicolumn{3}{|c|}{ Percentagens (\%) } \\
\hline Fragmentos de pelos de roedor & 9,0 & 30,0 & 61,0 \\
\hline Fragmentos de insetos & 21,0 & 65,0 & 14,0 \\
\hline Ácaros & 88,0 & 7,0 & 5,0 \\
\hline Bárbulas & 79,0 & - & 21,0 \\
\hline
\end{tabular}

Fonte: Elaborada pelos autores, 2019.

esse contaminante nas amostras adulteradas, contribuindo para a redução quantitativa dessa contaminação.

Conforme a RDC Anvisa $n^{\circ} 14 / 2014^{13}$, que estabelece para a páprica o limite de 80 fragmentos de insetos, 11 fragmentos de pelos de roedor e cinco ácaros na alíquota analítica de $25 \mathrm{~g}$, $77,0 \%$ das amostras foram consideradas insatisfatórias por apresentarem matérias estranhas acima do limite tolerado.

No total das amostras analisadas, $88,0 \%$ foram consideradas insatisfatórias segundo a RDC Anvisa n ${ }^{\circ} 14 / 2014^{13}$ ou a RDC Anvisa n ${ }^{\circ} 259 / 2002^{12}$, sendo que $19,0 \%$ estavam em desacordo com ambos os regulamentos.

Entre as amostras insatisfatórias, $61,0 \%$ apresentaram fragmentos de pelo de roedor acima do limite tolerado pela legislação, porém, dentre as que excederam o limite para fragmentos de insetos $(14,0 \%)$, nenhuma continha fragmentos de pelos de roedor acima do limite, concomitantemente. Não há limites na legislação para a presença de bárbulas em páprica, o que implica que as amostras que apresentaram essa matéria estranha $(21,0 \%)$ foram consideradas insatisfatórias, independente da quantidade encontrada (Tabela 2).

A legislação brasileira para matérias estranhas é semelhante à norma regulamentadora dos Estados Unidos quanto ao limite de fragmentos de insetos e de pelos de roedor para páprica. A Food and Drug Administration preconiza que devem ser apreendidos os lotes de páprica que apresentem mais que 75 fragmentos de insetos em $25 \mathrm{~g}$ ou mais que 11 fragmentos de pelo de roedor nessa mesma alíquota, após análise conforme método 977.25B da $\mathrm{AOAC}^{23}$. Já em outros países esse limite é mais rígido, como é o caso de Malawi, no continente africano, onde a páprica deve estar livre de insetos (vivos ou mortos), fragmentos de insetos, contaminação por roedores e sem a presença de fungos visíveis ${ }^{24}$.

Presume-se, portanto, que a ocorrência de matérias estranhas nas pápricas analisadas está relacionada às precárias condições de higiene no processo de produção e/ou falhas das boas práticas, enquanto que as fraudes registradas são sugestivas de adição intencional de ingrediente, com objetivo de obtenção de vantagem econômica, não sendo possível aferir sobre em que etapa da cadeia produtiva esta prática ocorreu.

\section{CONCLUSÕES}

A análise histológica evidenciou adulterações fraudulentas nas pápricas analisadas por meio da adição de outros vegetais, principalmente milho e urucum, evidenciando a importância da adoção de medidas de controle e fiscalização para a prevenção de prejuízos econômicos e riscos à saúde do consumidor.

O elevado percentual de amostras de páprica insatisfatórias, principalmente pela presença de fragmentos de pelos de roedor, observado neste estudo indica a necessidade de revisão nas tecnologias de produção adotadas e de adoção de medidas que evitem ou minimizem essa contaminação.

Os resultados deste trabalho ressaltam a necessidade de intensificação da vigilância desse tipo de produto com vistas à garantia de sua oferta à população de forma fidedigna e segura.

\section{REFERÊNCIAS}

1. Governo do Estado de São Paulo. Decreto $N^{\circ} 12.486$, de 20 de outubro de 1978. Aprova normas técnicas especiais relativas a alimentos e bebidas. Diário Oficial Estado. 21 out 1978.

2. Alves LP. Crescimento e produção de pimentão, tipo páprica, sob diferentes níveis de adubação de nitrogênio e fósforo [dissertação]. Mossoró: Universidade Federal Rural do Semi-Árido; 2006.

3. Carvalho SIC, Bianchetti LB, Ribeiro CSC, Lopes CA. Pimentas do gênero Capsicum no Brasil. Brasília: Empresa Brasileira de Pesquisa Agropecuária; 2006.

4. Ribeiro CSC. Qualidade de páprica. In: Anais do $55^{\circ}$ Congresso Brasileiro de Oleiricultura; Salvador, Brasil. Recife: Associação Brasileira de Horticultura; 2012
5. Moore JC, Spink J, Lipp M. Development and application of a database of food ingredient fraud and economically motivated adulteration from 1980 to 2010. J Food Sci. 2012;77(4):118-26. https://doi.org/10.1111/j.1750-3841.2012.02657.x

6. Johnson R. Food fraud and economically motivated adulteration of food and food ingredients. Congressional Research Service. 10 jan 2014[acesso 5 set 2019]. Disponível em: https://fas.org/sgp/crs/misc/R43358.pdf

7. Székács A, Wilkonson MG, Mader A, Appel B. Environmental and food safety of spices and herbs along global food chains. Food Control. 2018;83:1-6. https://doi.org/10.1016/j.foodcont.2017.06.033 
8. Ministério da Indústria, Comércio Exterior e Serviços (BR). Comex Stat: portal para acesso gratuito às estatísticas de comércio exterior do Brasil. Brasília: Ministério da Indústria, Comércio Exterior e Serviços; 2018[acesso 10 dez 2018]. Disponível em: http://comexstat.mdic.gov.br/pt/home

9. Abreu LF, Silva PA, Araújo EAF, Carvalho AV. Efeito da secagem sobre as propriedades antioxidantes de pimenta vermelhas Capsicum annuum var annuum. In: Anais do $25^{\circ}$ Congresso Brasileiro de Ciência e Tecnologia de Alimentos; Gramado, Brasil. Brasília: Empresa Brasileira de Pesquisa de Agropecuária; 2016.

10. Maurya VK, Gothandam KM, Ranjan V, Shakya A, Pareek S. Effect of drying methods (microwave vacuum, freeze, hot air and sun drying) on physical, chemical and nutritional attributes of five pepper (Capsicum annuum var annuum) cultivars. J Sci Food Agric. 2018;98(9):3492-500. https://doi.org/10.1002/jsfa. 8868

11. Correia M, Daros VSMG, Silva RP. Matérias estranhas em canela em pó e páprica em pó, comercializadas no estado de São Paulo. Cienc Tecnol Aliment. 2000;20(3):375-80. https://doi.org/10.1590/\$0101-20612000000300016

12. Agência Nacional de Vigilância Sanitária - Anvisa. Resolução RDC No 259, de 20 de setembro de 2002. Aprova o regulamento técnico para rotulagem de alimentos embalados. Diário Oficial União. 23 set 2002.

13. Agência Nacional de Vigilância Sanitária - Anvisa. Resolução RDC $N^{\circ} 14$ de 28 de março de 2014. Dispõe sobre matérias estranhas macroscópicas e microscópicas em alimentos e bebidas, seus limites de tolerância e dá outras providências. Diário Oficial da União. 28 mar 2014.

14. Rodrigues MMS, Atui MB, Correia M. Métodos de análise microscópica de alimentos: isolamento de elementos histológicos. São Paulo: Letras \& Letras; 1999.

15. Oliveira F, Ritto JLA, Jorge LIF, Barroso ICE, Prado BW. Microscopia de alimentos: exames microscópicos de alimentos in natura e tecnologicamente processados. São Paulo: Atheneu; 2015.
16. Association of Official Analytical Collaboration (AOAC) International. Official methods of analysis of AOAC international. 20a ed. Rockville: Association of Official Analytical Collaboration (AOAC) International; 2016.

17. Teerink BJ. Hair of West-European mammals. Cambridge: Cambridge University; 1991.

18. Belmain S. Assessment of the impact of rodents on rural household food security and the development of ecologically-based rodent management strategies in Zambézia province, Mozambique. London: Natural Resources Institute; 2002[acesso 18 ago 2019]. Disponível em: https: / /assets.publishing.service.gov.uk/ media/57a08d48ed915d3cfd001930/R7372_FTR.pdf

19. Atui MB, Lázzari FA, Zamboni CQ. Efeito do processamento do milho em grão no nível de matérias estranhas encontradas no grits e fubá. Rev Inst Adolfo Lutz.1998;57(1):57-63.

20. Dupchak LM. Detecção de sujidades e avaliação de uma formulação de pós inertes para o controle de inseto em grãos e farinhas de trigo [dissertação]. Curitiba: Universidade Federal do Paraná; 1997.

21. Gorham JR. Ecology and management of food-industry pests. Arlington: Association of Official Analytical Chemists; 1991.

22. Graciano RAS, Atui MB, Dimov MN. Avaliação das condições higiênico-sanitárias de cominho e pimenta do reino em pó comercializados em cidades do estado de São Paulo, Brasil, mediante a presença de matérias estranhas. Rev Inst Adolfo Lutz. 2006;65(3):204-8.

23. US Food and Drug Administration - FDA. Capsicum pods, ground capsicums excluding paprika, ground paprika: adulteration with insect and rodent filth, mold, mammalian excreta. Silver Spring: US Food and Drug Administration; 2018[acesso 16 ago 2019]. Disponível em: https://www.fda.gov/media/71842/download

24. Malawi Bureau of Standards - MBS. Ground paprika (Capsicum annuum L): specification. Malawi: Malawi Bureau of Standards; 2016[acesso 16 ago 2019]. Disponível em: https://docs.wto.org/dol2fe/Pages/FE_Search/ExportFile. aspx?id=239083\&filename=2017/TBT/MWI/17_4371_00_e.pdf

\section{Agradecimentos}

Ao Antonio Roberto de Souza Ferreira, técnico de apoio à pesquisa do Centro de Alimentos do Instituto Adolfo Lutz Central, pelos registros fotográficos realizados. Ao Hilberto Matosalém de Souza, auxiliar de apoio à pesquisa do Centro de Alimentos do Instituto Adolfo Lutz Central, pelo processamento das amostras.

Contribuição dos autores

Yamada LFP, Nogueira MD, Aquino Cl, Silva AM, Mattos EC - Concepção e planejamento (desenho do estudo), processamento das amostras, análise e interpretação dos dados e redação do trabalho. Marciano MAM - Concepção, planejamento (desenho do estudo), análise dos dados e redação do trabalho. Todas as autoras aprovaram a versão final do trabalho.

\section{Conflito de Interesse}

Os autores informam não haver qualquer potencial conflito de interesse com pares e instituições, políticos ou financeiros deste estudo.

Licença CC BY-NC atribuição não comercial. Com essa licença é permitido acessar, baixar (download), copiar, imprimir, compartilhar, reutilizar e distribuir os artigos, desde que para uso não comercial e com a citação da fonte, conferindo os devidos créditos de autoria e menção à Visa em Debate. Nesses casos, nenhuma permissão é necessária por parte dos autores ou dos editores. 